\title{
Synthesis of gallic acid imprinted polymer and incorporation in poly(caprolactone) mat via electrospinning
}

\author{
J. D. P. Moraes Segundo ${ }^{1 *}$, M. O. S. Moraes $^{2}$, W. R. Brito ${ }^{2}$, K. Segala ${ }^{2}$, Y. Garcia-Basabe , $^{3}$ \\ M. L. M. Rocco ${ }^{4}$, M. A. d'Ávila ${ }^{1}$ \\ ${ }^{1}$ Department of Manufacturing and Materials Engineering, Faculty of Mechanical Engineering, University of Campinas, \\ Campinas, 13083-860 São Paulo, Brazil \\ ${ }^{2}$ Department of Chemistry, Institute of Exact Sciences, Federal University of Amazonas, 69067-005 Manaus, Amazonas, \\ Brazil \\ ${ }^{3}$ Institute of Science of Life and Nature - ILACVN, Federal University of Latin-American Integration, 85866-000 Foz do \\ Iguaçu, PR, Brazil \\ ${ }^{4}$ Institute of Chemistry, Federal University of Rio de Janeiro, Rio de Janeiro, 21941-901 Rio de Janeiro, Brazil
}

Received 28 October 2020; accepted in revised form 21 January 2021

\begin{abstract}
Molecularly imprinted polymers (MIP) are materials capable of recognizing specific molecules, usually through non-covalent bonds. In this work, gallic acid (GA) was selected as the imprinting molecule, and MIP for GA (MIP-GA) was synthesized by bulk polymerization. X-ray photoelectron spectroscopy (XPS) and near-edge X-ray absorption fine structure (NEXAFS) confirmed the MIP-GA recognition property. Poly(caprolactone) mat containing MIP-GA particles (MIM-GA) was produced by electrospinning, which was morphologically characterized by Scanning electron microscopy (SEM). Functionalized MIM-GA was obtained by washes sequence in methanol for the GA removal from MIM-GA. The GA removal was confirmed by High-performance liquid chromatography (HPLC) with UV-Vis detection at 214 and $268 \mathrm{~nm}$. The functionalized MIM-GA immersed in GA methanolic solution with a concentration of $1.0 \mathrm{mM}$ extracted $96 \pm 1.5 \%$ of GA at $t=$ 150 minutes. The vibrational modes obtained from FT-IR spectra confirmed the chemical composition of MIM-GA and its functionalization through of band at $770 \mathrm{~cm}^{-1}$. The contact angle measurements were $134.0 \pm 1.0^{\circ}$ for electrospun MIM-GA and $125.0 \pm 1.2^{\circ}$ for functionalized MIM-GA. The decrease was due to the absence of GA after the functionalization process. The produced MIM-GA by electrospinning acquired the recognition property of the GA imprinted polymer synthesized, which can be used for the specific detection, extraction, and purification of this important natural polyphenol.
\end{abstract}

Keywords: polymer membranes, electrospinning, poly(caprolactone), gallic acid

\section{Introduction}

Molecularly imprinted polymers (MIP) are materials capable of recognizing specific molecules, usually through non-covalent bonds. Their development has attracted considerable interest in the scientific community due to their potential application in the filtration, chromatography, and sensors field [1]. Besides, attractive alternatives have been reported in the literature to develop MIP for specific applications, such as electrospinning [2], nanostructured [3], and MIP scaffold [4].

MIPs are synthesized through different methods, and depending on the final application of the product, different physical forms can be obtained [5]. The main components for the MIPs synthesis are a functional monomer, standard molecule, cross-linker agent, initiator, and solvent [6]. Generally, the methods occur through free radical polymerization mechanism, 
including mass, suspension, emulsion, precipitation polymerizations, etc. [5, 7]. After synthesis, the removal of the standard molecule from the polymeric matrix, usually by washing with specific solvents, results in the material functionality, thus conferring the presence of active recognition sites [8].

Porous materials and membranes with selective properties capable of trapping and recognizing specific molecules are produced by phase inversion [9], assisted by water [10], functionalization [11], electrospray [12], and other methods [1, 14], including the electrospinning [13] which is an interesting technique to produce fibrous nonwoven mats.

Electrospinning produces polymeric fibers in micrometric, sub-micrometric, and nanometric dimensions [15]. It has recently been used to develop molecularly imprinted membranes (MIM) with desirable molecule recognition [13, 15-18]. Yoshimatsu et al. [16] produced MIP particles loaded on an electrospun mat to obtain a MIM to extract propranolol from an aqueous solution. Liu et al. [17] reported the MIP addition to the PET electrospun membrane for rhodamine $\mathrm{B}$ detection. Besides, polystyrene nanofiber-based MIP was obtained via electrospinning to produce MIM for pesticide absorption was reported [13].

Gallic Acid (GA) is a natural polyphenol found in various plants [19]. This substance and its derivatives are organic compounds of a broad interest in the pharmaceutical industry and exhibit excellent therapeutic properties such as antitumor, antimicrobial, antifungal, anticancer, and antiviral. Moreover, it is also known as an excellent antioxidant $[19,20]$. In the context mentioned above about MIP, the development of new alternatives for the detection, extraction, and purification of polyphenols is attractive to the pharmaceutical, cosmetic, biotechnological, and biomedical industries [21]. Pardeshi and collaborators widely studied the GA to obtain MIP of GA (MIP-GA) to extract phenolic compounds [22, 23]. Chuysinuan et al. [19] synthesized polymeric fibers of poly(L-lactic acid) (PLLA) by electrospinning containing GA. The material presented great potential for use in the transdermal and topical release of the substance. Pardeshi and researchers [22] developed particles and molecularly imprinted microspheres of GA for selective extraction of this substance from plant extract. Moreover, Croitoru and Patachia [24] produced the GA imprinted film using polyvagentyinyl alcohol (PVA), and the results showed a good selectivity of the molecule.
This work aimed to demonstrate the production, characterization, and functionalization of electrospun MIM-GA. For that, GA imprinted polymer was synthesized, and its recognition property was confirmed by X-ray photoelectron spectroscopy (XPS) and nearedge X-ray absorption fine structure (NEXAFS).

\section{Experimental}

\subsection{Material}

Ethyleneglycoldimethacrylate (EGDMA, 98\%, 335681, Aldrich, Germany), 2,2'-Azobis (2-methylpropionitrile) (AIBN, 98\%, 714887, Aldrich, Germany), acetonitrile (ACN, 99.8\%, 271004, Aldrich, Germany), acrylamide (99\%, A8887, Aldrich, Germany), gallic acid (GA, $M M=170.12 \mathrm{~g} / \mathrm{mol}$, V900107, Aldrich, Germany), chloroform (99.5\%, 1024451000, Merck, Germany), acetone (99.5\%, A101701BJ, Synth, Brazil), methanol (99.8\%, 00495, Neon, Brazil), and poly (caprolactone) (PCL, $M M=$ 70000-90000 g/mol, 1001080125, Aldrich, USA). Deionized water (electrical conductivity $=$ $\left.0.5 \mu \mathrm{S} / \mathrm{cm}^{2}\right)$.

\subsection{Gallic acid imprinted polymer synthesis}

The GA imprinted polymer synthesis by bulk polymerization was performed on methods described in the literature [23]. Firstly, into a flask were added GA $(17 \mathrm{mg})$, functional acrylamide monomer $(28 \mathrm{mg})$, EGDMA as the cross-linker (7.5 ml), AIBN (1 ml), and acetonitrile as porogenic solvent $(10 \mathrm{ml})$. Afterward, the flask with the substances was conducted in an ultrasound bath for 15 minutes. The polymerization solution was purged with inert gas (nitrogen) for 5 minutes in the ice bath. Then, the flask was closed and immersed in a water bath for 4 hours at $60^{\circ} \mathrm{C}$. The synthesized GA imprinted polymer was grounded with a mortar for 1 hour to obtain particles.

The non-imprinted polymer (poly (acrylamide)) was synthesized using the same method; however, without the addition of the GA, which was used for comparative study in the spectroscopic characterization of materials chemical composition.

\subsection{Preparation of PCL solution and production of MIM-GA via electrospinning}

PCL solution was prepared according to the literature $[15,25]$. Firstly, the acetone and chloroform mixture (1:1 mass ratio) dissolved $1 \mathrm{~g}$ PCL with mechanical stirring at room temperature for 4 hours. 
Then, $250 \mathrm{mg}$ MIP-GA particles were added into the PCL solution with mechanical stirring for 1 hour until the complete dispersion of the particles.

The mixture solution has transferred into a syringe with a metallic needle (21Gx 1 "), and the electrospinning system has operated under the following conditions: applied voltage of $12 \mathrm{kV}$; flow rate of $8 \mathrm{ml} / \mathrm{h}$; rectangular metal collector type; tip-to-collector distance was $17 \mathrm{~cm}$, and environmental parameters, such as humidity and temperature were approximately $56 \%$ and $23^{\circ} \mathrm{C}$, respectively.

Electrospun fibers were deposited on the collector to form the PCL mat containing gallic acid imprinted polymer particles (MIM-GA). Electrospun PCL mat has been produced with the same parameters but without the presence of MIP-GA particles. All materials provided were vacuum dried to remove volatile impurities.

\subsection{Functionalization process}

Figure 1 shows the functionalization process experimental scheme after the production of electrospun MIM-GA adapted from the literature description [22, 23] to attribute the GA recognition property to the electrospun MIM-GA and GA imprinted polymer.

The MIM-GA was immersed in a glass flask containing $5 \mathrm{ml}$ of methanol and conducted in the ultrasound bath for 5 minutes. Subsequently, the MIM-GA was removed and immersed in another glass flask containing $5 \mathrm{ml}$ of methanol and subjected to an ultrasound bath again. This wash (elution) sequence occurred four times. An aliquot of $1 \mathrm{ml}$ of each wash was collected and analyzed by HPLC (Thermo Scientific - Model Finnigan Surveyor), equipped with a diode array detector (DAD), in the ultraviolet-visible (UV-vis) region with a range of $200-800 \mathrm{~nm}$. The measurements were performed in triplicate, and the reference absorption bands of GA were at 214 and $268 \mathrm{~nm}$. The same wash procedure was used in GA imprinted polymer, except in the XPS and NEXAFS techniques described in Sections 2.5 and 2.6, respectively, that were used to confirm its GA recognition property.

\subsection{Characterization by X-ray photoelectron spectroscopy (XPS)}

The recognition capacity of the GA imprinted polymer was evaluated by XPS (Thermo Scientific Model ESCALAB 250Xi), equipped with an electron energy hemispherical analyzer and using as an
$\mathrm{X}$-ray source the monochromatized $\mathrm{Al} \mathrm{K} \alpha$ line $(h v=$ $1486.6 \mathrm{eV})$.

Films of GA imprinted polymer were analyzed in its central region $($ spot size $=650 \mu \mathrm{m})$. Survey spectra were measured from 0 to $1300 \mathrm{eV}$ with a pass energy of $50 \mathrm{eV}$, and high-resolution spectra acquired with $25 \mathrm{eV}$ pass energy at $\mathrm{C} 1 \mathrm{~s}, \mathrm{~N} 1 \mathrm{~s}$, and $\mathrm{O} 1 \mathrm{~s}$ core levels. The binding energy scale was calibrated using the Au $4 \mathrm{f}_{7 / 2}$ signal. The sensitivity factors were used to determine each atomic concentrations of elements in the samples [26].

\subsection{Characterization by near edge $X$-ray absorption fine structure (NEXAFS)}

NEXAFS was also employed to evaluate the recognition capacity of the GA imprinted polymer. GA imprinted polymer films were analyzed at the spherical grating monochromator (SGM) beamline (250$1000 \mathrm{eV}$ ) of the Brazilian Synchrotron Light Laboratory (LNLS). NEXAFS spectra were obtained in the total electron yield (TEY) mode at the K absorption edge of carbon and oxygen. The spectra were normalized by the incident photon flux $\left(I_{0}\right)$, measuring the electron drain current in an Au grid. All measurements were performed in a high vacuum chamber $\left(10^{-8} \mathrm{mbar}\right)$. Phenylalanine sample was used as a standard reference for photon energy calibration for the NEXAFS spectra [27].

\subsection{Morphological characterization}

The GA imprinted polymer particles, electrospun MIM-GA, and electrospun PCL mats were characterized using a Scanning electron microscope - SEM (CARL ZEISS - Model LEO 435 VP). All samples were coated with gold sputtering (BAL TEC - Model CPD 050). Software ImageJ (free version) [28] was used for particle size analysis and fiber diameter measurements from the SEM images. The values were presented as the mean values and standard deviations (mean $\pm \mathrm{SD}$ ) using methods described in the literature [25].

\subsection{Analysis by high-performance liquid chromatography (HPLC)}

GA calibration curve was performed using GA in methanol [23] with concentrations in the range of $3.9-100 \mu \mathrm{g} / \mathrm{ml}$. HPLC/UV-vis was used in the range of $200-800 \mathrm{~nm}$. The GA was identified on 214, and $268 \mathrm{~nm}$, and a calibration curve obtained was $y=$ $5.200 x+0.024\left(R^{2}=0.993\right)$, where " $y$ " represents 
the absorbance, " $x$ " the concentration, 5.200 and 0.024 are constants. This equation was used to calculate unknown concentrations in the extraction process described following.

GA extraction process using the functionalized MIM-GA was performed on dimensions $18 \mathrm{~mm} \times$ $8 \mathrm{~mm}$ and weighed $m=11 \mathrm{mg}$. It was immersed into the flask with GA methanolic solution with $C=$ $1.0 \mathrm{mM}$. The concentration was monitored in the times $0,30,60,90,120,150$, and 180 minutes. The concentration values were measured using the calibration curve and inserted in Equation (1) to calculate the GA extraction in percentage [\%]. All the measurements were obtained in triplicate:

GA extraction $=\frac{C_{\mathrm{i}}-C_{\mathrm{f}}}{C_{\mathrm{f}}} \cdot 100[\%]$

where $C_{\mathrm{i}}$ and $C_{\mathrm{f}}$ are the initial concentrations and final concentrations [mM], respectively. The illustration of the production and performance of functionalized MIM-GA can be observed in Figure 1.

\subsection{FTIR spectroscopic characterization}

Synthesized GA imprinted polymer, GA, PCL, poly (acrylamide), electrospun PCL mat, electrospun MIM-GA, and functionalized MIM-GA were spectroscopically characterized by FTIR (NICOLET Model IR 200) in the $400-4000 \mathrm{~cm}^{-1}$ range, with

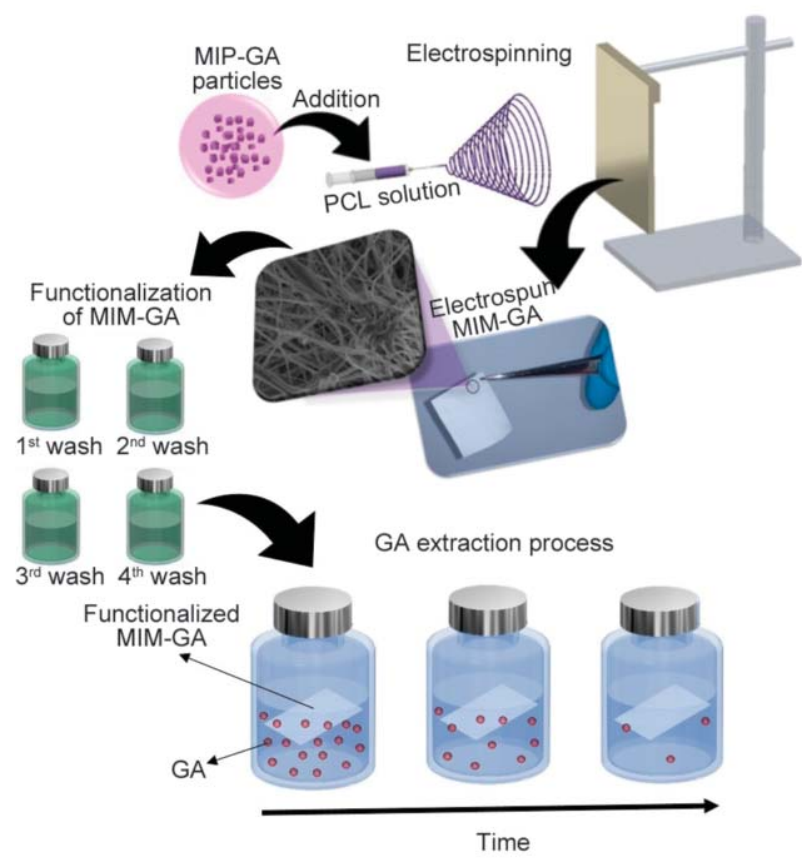

Figure 1. Experimental scheme of the production, functionalization, and GA extraction process of MIM-GA produced by electrospinning.
32 scans with a spectral resolution of $4 \mathrm{~cm}^{-1}$. The samples were mixed in the 1:100 ratio with potassium bromide $(\mathrm{KBr})$ and manually pressed by $80 \mathrm{kN}$ for 2 minutes.

\subsection{Contact angle determination}

Contact angle measurements were obtained by applying a $10 \mu 1$ drop of deionized water on the electrospun PCL mat, electrospun MIM-GA, and functionalized MIM-GA surfaces. The drop behavior was observed with a digital microscope (DINO - Lite plus, $1000 \times$ resolution) for 120 seconds at room temperature. The Drop Analysis - LB-ADSA plugin was installed in ImageJ software, and the Low Shape Axisymmetric Drop Shape Analysis tool was used. The contact angle value was measured manually in triplicate.

\section{Results and discussion}

\subsection{Morphological characterization}

Figure 2a shows SEM images of the morphological characteristics of MIP-GA particles with heterogeneous dispersion and an irregular shape with dimensions range from 1.1 to $25.4 \mu \mathrm{m}$. These characteristics are common in MIP synthesis by bulk polymerization [24].

SEM images of electrospun PCL mat and electrospun MIM-GA are shown in Figures $2 b$ and $2 c$, where their average diameter was $4.5 \pm 1.8$ and $4.0 \pm 1.6 \mu \mathrm{m}$, respectively. For both cases, the fibers presented a random orientation. Moreover, Figure $2 \mathrm{c}$ shows the presence of MIP-GA particles in the MIM-GA and fibers with decreased diameters.

The functionalization process carried out in the electrospun MIM-GA, described in Section 2.4, did not affect the integrity of the fiber that forms the porous structure of the electrospun MIM-GA. This occurred because the PCL is insoluble in methanol. Figure $2 \mathrm{~d}$ shows an experimental test of MIM-GA in methanol, and Figure 2e shows the morphology of the functionalized MIM-GA after the functionalization process, which will be discussed later.

\subsection{GA removal from GA imprinted polymer analysis by XPS and NEXAFS}

Figure 3 shows C1s XPS spectra before and after the functionalization process of GA imprinted polymer. The peak at $284.8 \mathrm{eV}$ corresponds to the $\sigma$-bonds of aliphatic carbon atoms present in the polymeric chain $(-\mathrm{C}-\mathrm{C}-)$, the peak at $286.4 \mathrm{eV}$ is related to the 


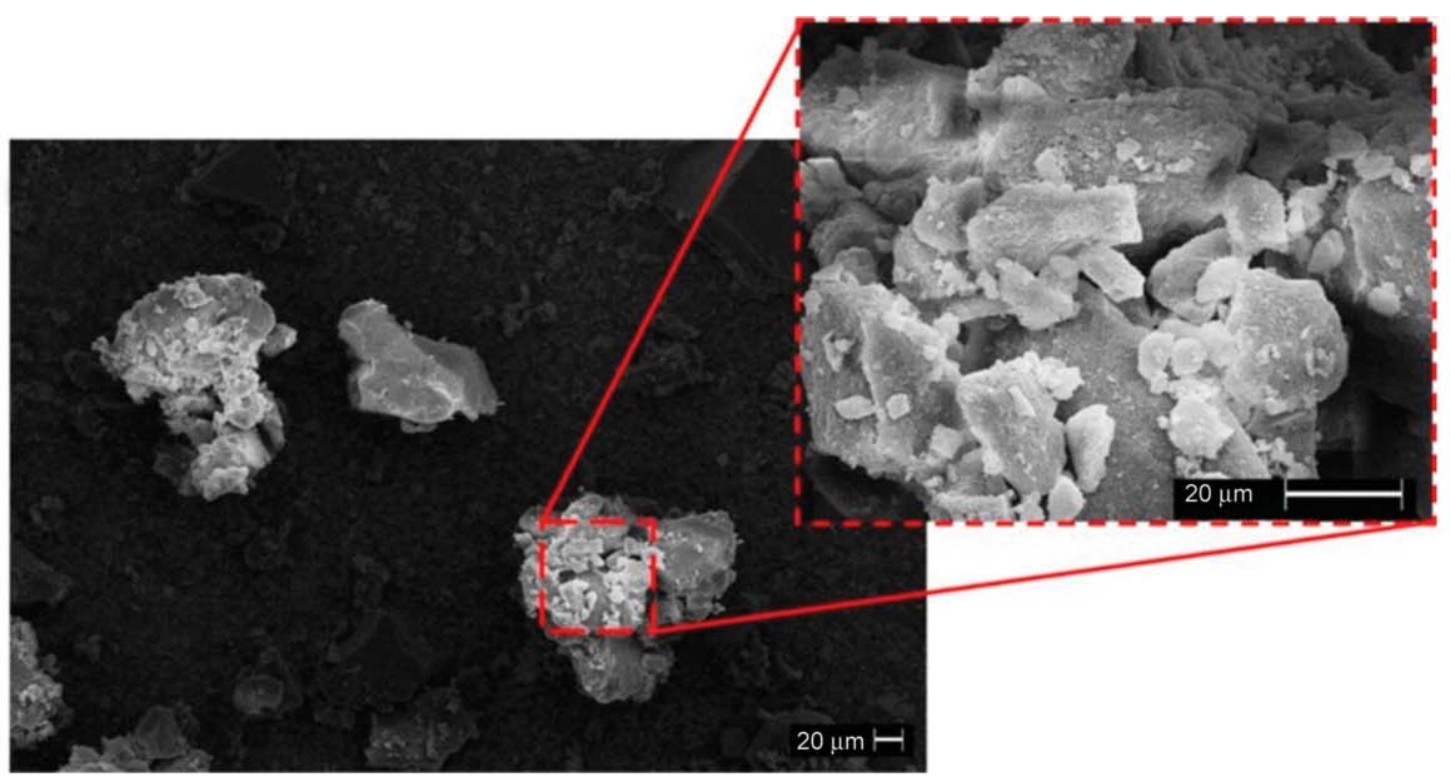

a)

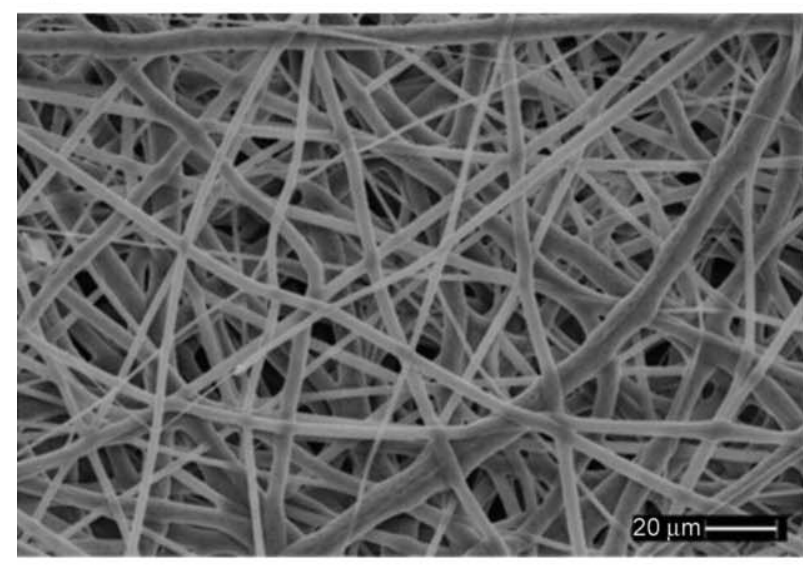

b)

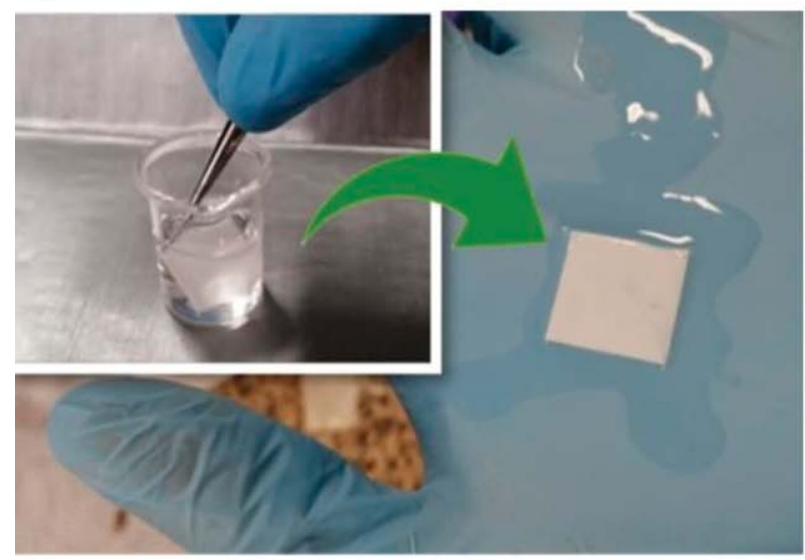

d)

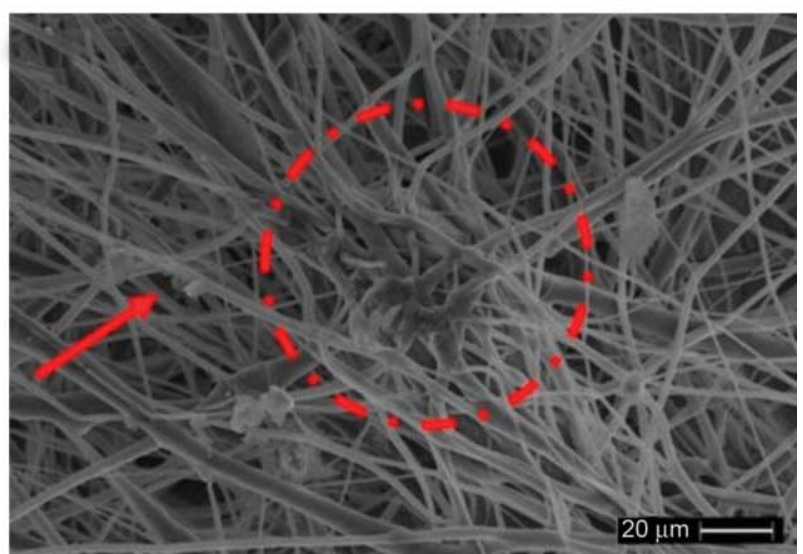

c)

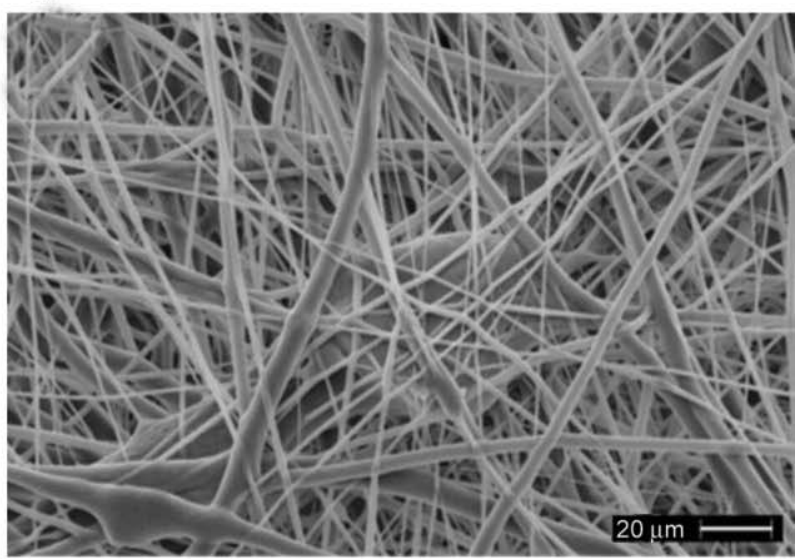

e)

Figure 2. SEM images of GA imprinted polymer (a), electrospun PCL mat (b), electrospun MIM-GA (c), MIM-GA after washing in methanol (d), and functionalized MIM-GA (e).

$\mathrm{C}-\mathrm{N}$ and $\mathrm{C}-\mathrm{O}$ bonds, and the peak at $288.8 \mathrm{eV}$ attributed to carbonyl $(\mathrm{C}=\mathrm{O})$ [29-32].

The decrease in the intensity of peaks at positions 286.4 and $288.8 \mathrm{eV}$ is due to GA removal from GA imprinted polymer after the functionalization process, which corresponds to $\mathrm{C}-\mathrm{O} / \mathrm{C}-\mathrm{N}$ and $\mathrm{C}=\mathrm{O}$ bonds, respectively.

Binding energies of $\mathrm{C}-\mathrm{N}$ (from the amide group of the polymeric matrix) and $\mathrm{C}-\mathrm{O}$ (from the phenolic group of GA) are close without causing any shift. 


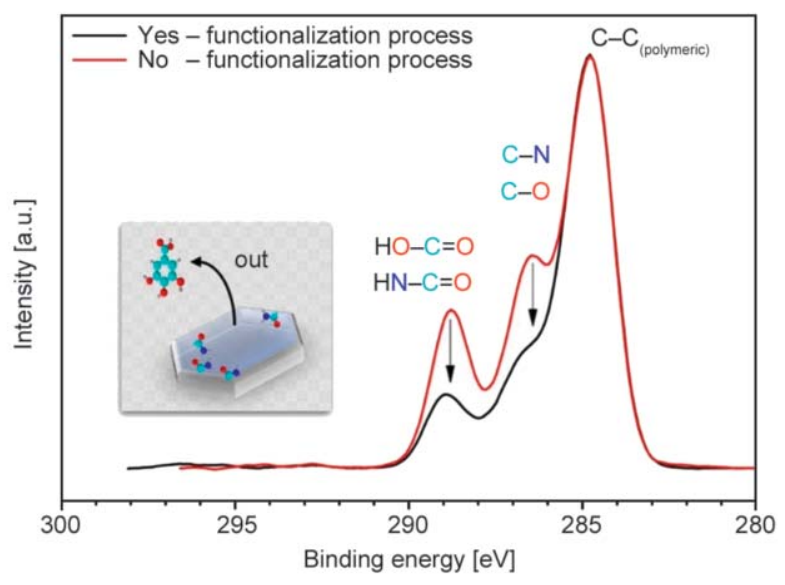

Figure 3. C1s XPS spectra of GA imprinted polymer before and after the functionalization process. Inset: the illustrative diagram shows the output of the GA, reflecting the decrease of the corresponding peaks (red line).

However, after the GA removal, there is a decrease in intensity at $286.4 \mathrm{eV}$. The same is observed for the peak at $288.8 \mathrm{eV}$ associated with the carbonyl present in the amide molecular group $\left(\mathrm{O}=\mathrm{C}-\mathrm{NH}_{2}\right)$ of the polymeric matrix and the carboxylic acid group $(\mathrm{O}=\mathrm{COH})$ of gallic acid.

The interaction between the GA molecules and the active cavities is of the H-bond type. After the GA removal process (functionalization process), a decrease in the intensity of the peaks in the $\mathrm{C} 1 \mathrm{~s}$ spectrum related to $\mathrm{HO}-\mathrm{C}=\mathrm{O}, \mathrm{HN}-\mathrm{C}=\mathrm{O}, \mathrm{C}-\mathrm{N}$, and $\mathrm{C}-\mathrm{O}$ molecular groups was observed. Functionalization occurs when the amide molecular groups of the polyacrylamide are free after GA removal. The decrease in the intensity of the peaks is related to the output of the gallic acid molecules that have the greatest contribution to the molecular groups of carboxylic acid and hydroxyls. Figure 4 shows the high-resolution XPS spectra at O1s core level of GA imprinted polymers before and after the functionalization process. The experimental data (black line) was deconvoluted by two Gaussian curves for each spectrum. The first peak centered at $532.2 \mathrm{eV}$ is attributed to carbonyl $(\mathrm{C}=\mathrm{O})$, certainly present in the gallic acid and the polymeric matrix (polyacrylamide) [32]. The second peak centered at $533.3 \mathrm{eV}$ is assigned to $\mathrm{C}-\mathrm{O}$ species [32].

The decrease in the intensity of peak at $533.3 \mathrm{eV}$ suggests that the intermolecular interactions between the amide group and the carboxylic acid group $(\mathrm{O}=\mathrm{C}-\mathrm{HN}-\mathrm{H} \cdots \mathrm{O}=\mathrm{C}-\mathrm{OH})$ and hydroxyls $(\mathrm{C}-\mathrm{OH})$ were ruptured after the use of a solvent in the process of removing the gallic acid molecules from the MIP

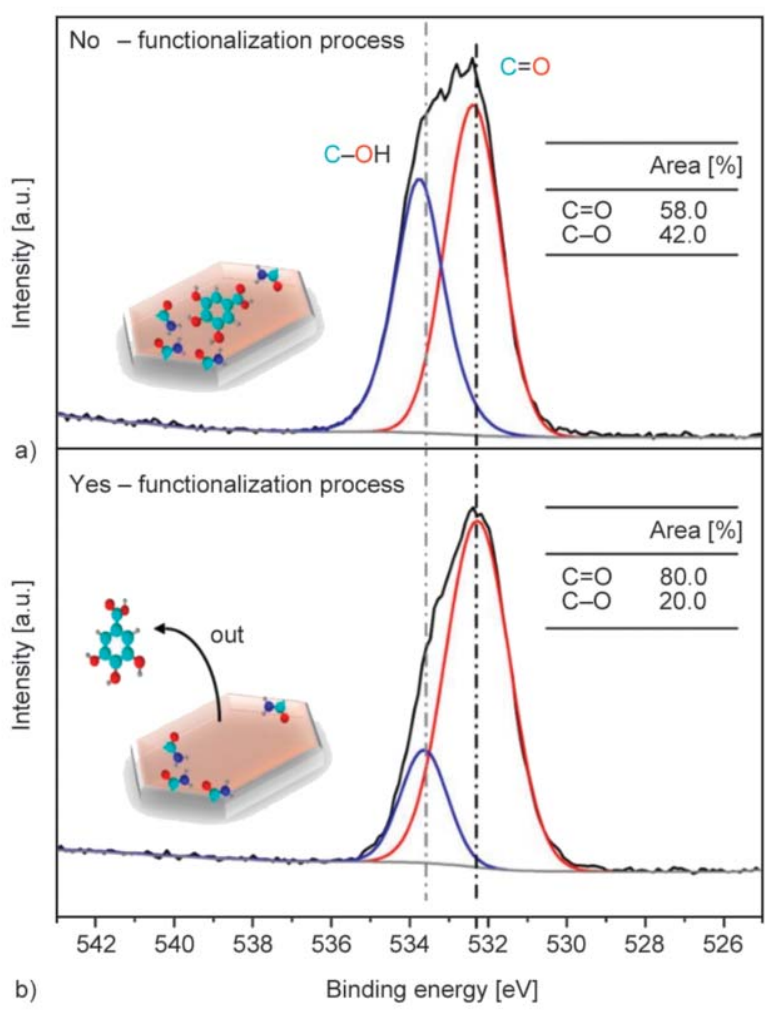

Figure 4. O1s XPS spectra of GA imprinted polymer before and after the functionalization process. Inset: illustrative scheme of imprinted polymer with GA (a) and imprinted polymer without GA (b), and Tables with percentage values of the area of each curve.

matrix. Therefore, the removal of GA molecules forms molecular cavities within the MIP formed by free amides, $\mathrm{O}=\mathrm{C}-\mathrm{NH}_{2}$, as shown in the scheme in Figure 4.

Another way of inferring the presence of free amides is through the percentage values of the area of the curves shown in the inset of Figure 4, where it is observed that for the GA imprinted polymer, the percentage of $\mathrm{C}=\mathrm{O}$ was equal to $58 \%$. In contrast, in the functionalized GA imprinted polymer, the $\mathrm{C}=\mathrm{O}$ percentage was $80 \%$, indicating a significant free amide presence.

Figure 5 shows the NEXAFS spectra at the C K-edge measured at normal incidence for GA imprinted polymer and functionalized GA imprinted polymer. Notably, there is a considerable contribution of the peak at $287.5 \mathrm{eV}$ associated with the electronic transition of $\mathrm{C} 1 \mathrm{~s} \rightarrow \pi^{*} \mathrm{C}=\mathrm{O}$ that corresponds to the carbonyl anti-bonding $\pi$ empty orbital [33-36]. Another observation was a shoulder in the region at $286.5 \mathrm{eV}$ associated with the electronic transition of $\mathrm{C} 1 \mathrm{~s} \rightarrow \pi^{*} \mathrm{C}=\mathrm{N}$ (green region) corresponding to tautomerism structures and amide- imidic acid resonance for the amide form in the polyacrylamide $[31,33,35,37]$. At 


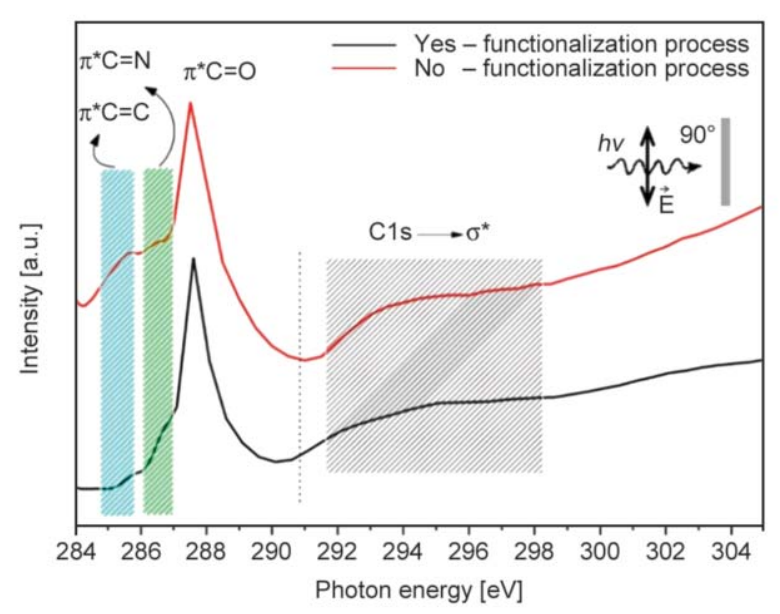

Figure 5. C K-edge NEXAFS spectra of GA imprinted polymer before and after the functionalization process.

$285.2 \mathrm{eV}$, there is a visible shoulder associated with the electronic transition $\mathrm{C} 1 \mathrm{~s} \rightarrow \pi^{*} \mathrm{C}=\mathrm{C}$ of the aromatic ring due to the presence of GA in the GA imprinted polymer (region highlighted in blue) $[33,35$, 38]. The broadband at higher photon energies, above the ionization potential, is assigned to transitions to $\sigma$-symmetry states $\left(\mathrm{C} 1 \mathrm{~s} \rightarrow \sigma^{*}\right)$.

Figure 6 shows the NEXAFS spectra at the oxygen K-edge. There are two peaks associated with $\mathrm{O} 1 \mathrm{~s} \rightarrow$ $\pi^{*} \mathrm{O}=\mathrm{C}-\mathrm{N}$ and $\mathrm{O} 1 \mathrm{~s} \rightarrow \pi^{*} \mathrm{O}-\mathrm{C}=\mathrm{N}$ electronic transitions at 530.1 and $532.5 \mathrm{eV}$, respectively, as a reflection of the tautomerism of the amide group $[35,36]$. The broad bands at higher photon energies correspond to transitions to $\sigma$-symmetry states $\left(\mathrm{O} 1 \mathrm{~s} \rightarrow \sigma^{*}\right)$. The spectra show high similarity in form and intensity. No relevant change was observed with the presence and absence of the gallic acid molecules.

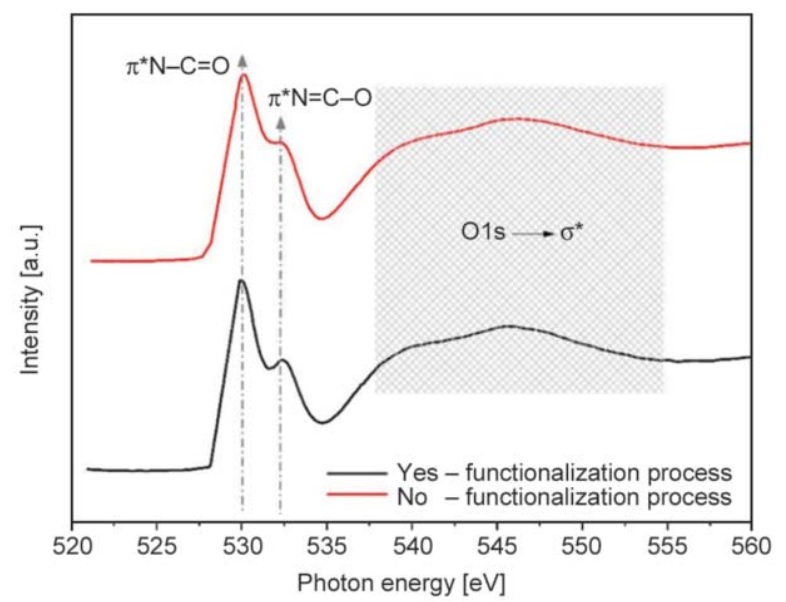

Figure 6. O K-edge NEXAFS spectra of GA imprinted polymer before and after the functionalization process.

\subsection{MIM-GA functionalization and GA extraction process}

The absorption spectra obtained by HPLC of the functionalization process are showed in Figure 7 and were compared with the absorption spectra of the GA solution in methanol with a concentration of $1.0 \mathrm{mM}$. The MIM-GA functionalization is a process that involves the superficial removal of GA molecules from the active sites present in electrospun MIM-GA, thus releasing active sites generated during molecular imprinting of MIP-GA particles, as was mentioned and discussed in Section 3.2 by XPS and NEXAFS.

As shown in Figure 7, the decrease in the band intensity at 214 and $268 \mathrm{~nm}$ is attributed to the subsequent removal of GA due to the MIM-GA washing sequence in methanol [23]. This result proves that the removal of GA from the electrospun MIM-GA occurred and, consequently, the MIM-GA functionalization. Thus, the functionalization process confers for functionalized MIM-GA the ability to recognize and retain GA molecules [22, 23].

The GA calibration curve is shown in Figure 8a and was used to calculate the concentration at different immersion times of the functionalized MIM-GA in the GA solution.

The percentage of GA extraction [\%] performed by the functionalized MIM-GA was calculated according to the literature using Equation (1) [39] and is showed in Figure 8b. We observed different values of GA extraction at the different interleaved times of 30 minutes. However, in all cases, the GA extraction was over $60 \%$, and, specifically, in immersion time in 150 minutes, the GA extraction achieved $96 \pm 1.5 \%$.

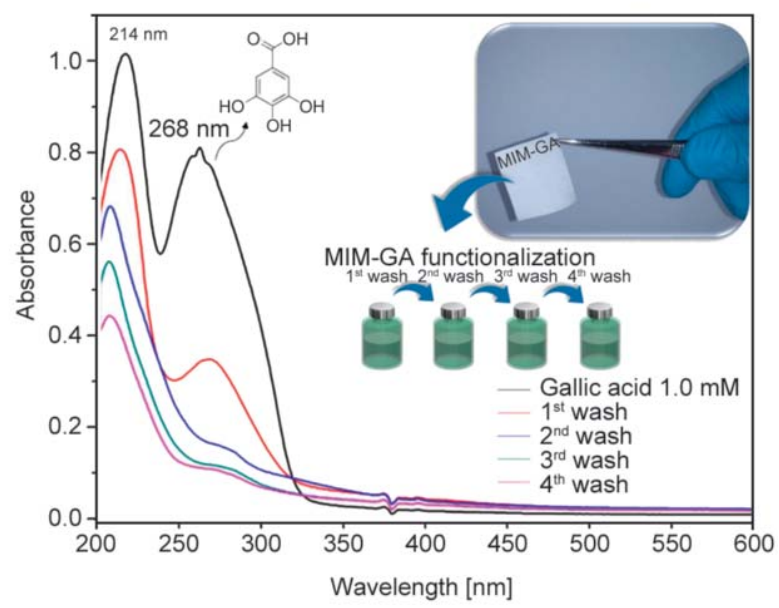

Figure 7. UV-vis spectra of methanolic solutions obtained from the functionalization process of MIM-GA using methanol solvent. 

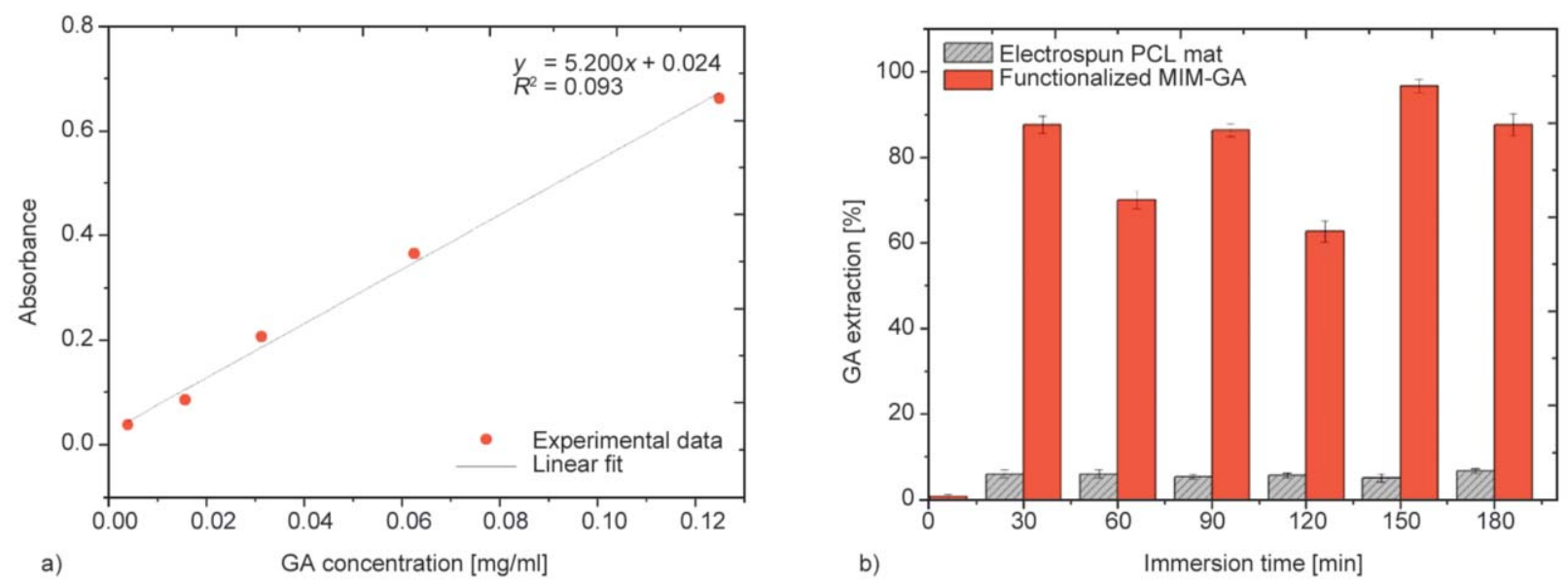

Figure 8. GA calibration curve (a) and percentage of GA extraction [\%] performed by the functionalized MIM-GA (b).

The electrospun PCL mat cannot extract the GA of the solution. It is important to highlight the values of GA extraction of the immersion times in $\mathrm{t}=30$ minutes for $87 \pm 2.0 \%, t=60$ minutes for $70 \pm 2.0 \%$, $t=90$ minutes for $86 \pm 1.5 \%, t=120$ minutes for $63 \pm 2.5 \%$ and $t=180$ minutes for $88 \pm 2.5 \%$. Despite these variations in GA extraction values, the MIMGA produced by electrospinning acquired the recognition property of GA imprinted polymer previously proven by the XPS and NEXAFS techniques.

\subsection{FT-IR spectroscopic characterization}

FTIR spectra of the electrospun MIM-GA (Figure 9 curve a), functionalized MIM-GA (Figure 9 curve b), electrospun PCL mat (Figure 9 curve c), GA (Figure 9 curve d), and poly (acrylamide) (Figure 9 curve e), this latter was mentioned in Section 2.2 as a non-imprinted polymer, is shown in Figure 9. The values of the major absorption bands of the samples are referenced [40-42] and listed in Table 1.

The absorption bands at $1731 \mathrm{~cm}^{-1}$ (carbonyl stretching), $1293 \mathrm{~cm}^{-1}$ (backbone $\mathrm{C}-\mathrm{O}$ and $\mathrm{C}-\mathrm{C}$ stretching modes), and $1238 \mathrm{~cm}^{-1}$ (asymmetric COC stretching) indicate the predominant presence of PCL as a support structure functionalized with GA imprinted polymer, as can be seen in Figure 2c [40].

FT-IR spectra of MIM-GA and functionalized MIMGA showed overlapping absorption bands such as in

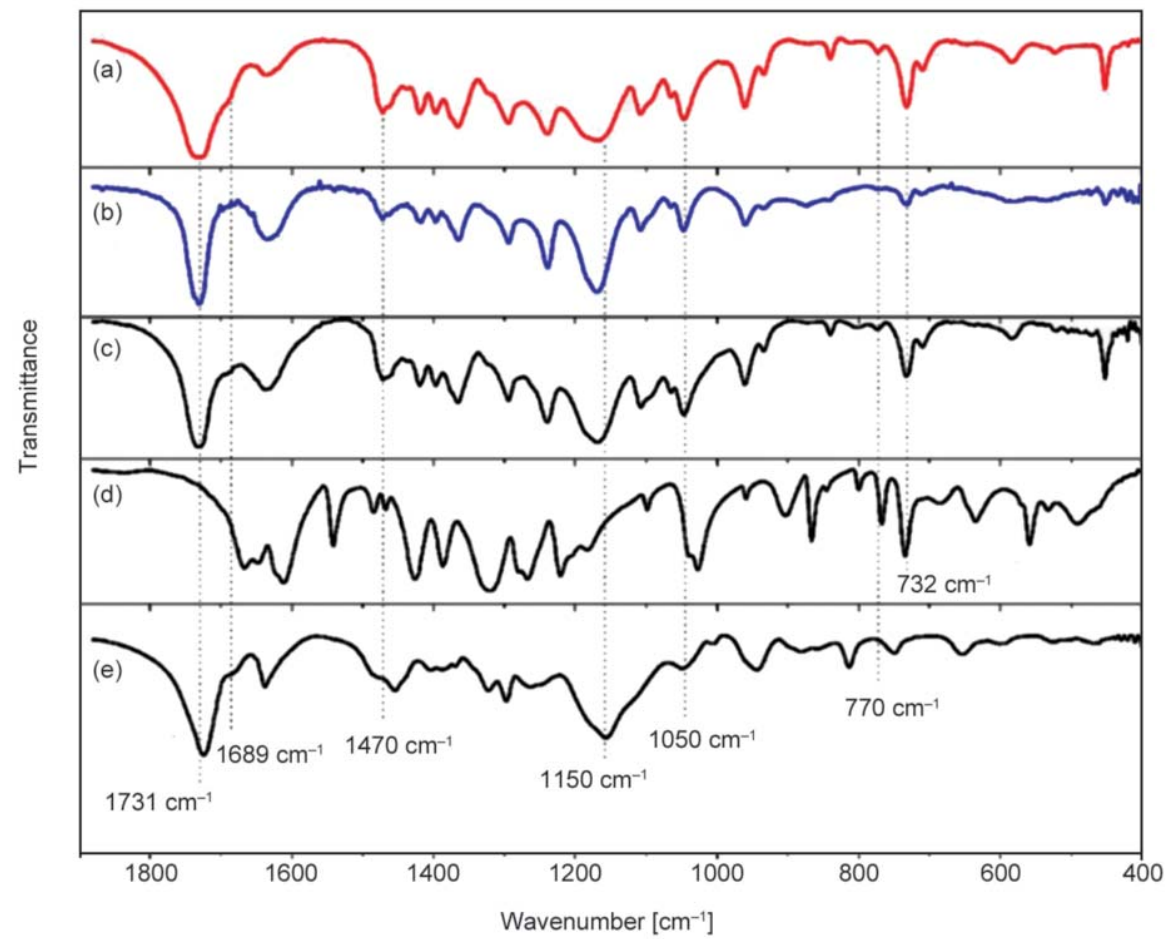

Figure 9. FTIR spectra of the electrospun MIM-GA (a) and functionalized MIM-GA (b) compared with electrospun PCL mat (c), GA (d), and poly(acrylamide) (e). 
Table 1. Absorption bands present in the FTIR spectra of the analyzed samples.

\begin{tabular}{|c|c|c|c|c|c|}
\hline \multirow{2}{*}{ Vibrational modes } & \multicolumn{5}{|c|}{$\begin{array}{c}\text { Wavenumber } \\
{\left[\mathrm{cm}^{-1}\right]}\end{array}$} \\
\hline & $\begin{array}{c}\text { Gallic Acid } \\
\text { [41] }\end{array}$ & $\begin{array}{c}\text { Poly(acrylamide) } \\
{[42]}\end{array}$ & \begin{tabular}{|c|} 
Electrospun PCL mat \\
{$[40]$}
\end{tabular} & $\begin{array}{c}\text { Functionalized } \\
\text { MIM-GA }\end{array}$ & $\begin{array}{c}\text { Electrospun MIM- } \\
\text { GA }\end{array}$ \\
\hline$v(C=O)$ stretching & - & $\begin{array}{l}1726 \\
1689\end{array}$ & 1731 & 1732 & $\begin{array}{l}1731 \\
1688\end{array}$ \\
\hline$v(C=C)$ stretching & $\begin{array}{l}1609 \\
1546\end{array}$ & - & - & - & - \\
\hline$v(C-O)$ stretching & $\begin{array}{l}1428 \\
1265 \\
1037\end{array}$ & 1044 & $\begin{array}{l}1293 \\
1238 \\
1049\end{array}$ & 1047 & 1047 \\
\hline$v(\mathrm{C}-\mathrm{N})$ stretching & - & $\begin{array}{l}1454 \\
1154\end{array}$ & - & 1169 & 1171 \\
\hline$-\left(\mathrm{CH}_{2}\right)$ n stretching & - & $\begin{array}{r}1453 \\
750\end{array}$ & $\begin{array}{r}1466 \\
733 \\
\end{array}$ & $\begin{array}{r}1470 \\
734 \\
\end{array}$ & $\begin{array}{r}1472 \\
732 \\
\end{array}$ \\
\hline Aromatic ring & $\begin{array}{l}768 \\
733\end{array}$ & - & - & 774 & 773 \\
\hline
\end{tabular}

$1731 \mathrm{~cm}^{-1}$ attributed to amide $\mathrm{C}=\mathrm{O}$ stretch from $\mathrm{p}$ NIP and to the ketone $\mathrm{C}=\mathrm{O}$ stretch from $\mathrm{PCL}$ and at $1050 \mathrm{~cm}^{-1}$ attributed to the vibration of the $\mathrm{C}-\mathrm{O}$ bond present in electrospun PCL mat, GA, and poly (acrylamide).

After the functionalization process of electrospun MIM-GA, the GA removal can be identified by the absence of the $770 \mathrm{~cm}^{-1}$ band corresponding to the aromatic vibrational mode present in GA (see functionalized MIM-GA spectrum in Figure 8). This result was expected since in Figure 7, it was shown through bands at 214 and $268 \mathrm{~nm}$ to remove GA by washes in methanol.

These results suggest the functionalization of MIMGA after the removal of GA molecules, increasing the number of active cavities where hydrogen bonding interactions occur [22]. Therefore, through these results, we believe that the functionalized MIM-GA acquired recognition property of GA imprinted polymer is confirmed by XPS and NEXAFS.

\section{Contact angle determination}

Figure 10 presents the graph of contact angle measurements and wettability that show the influence of GA in the electrospun MIM-GA and functionalized MIM-GA. The electrospun PCL mat contact angle was $142.0 \pm 2.5^{\circ}$, attributed to its hydrophobic behavior. This result was similar to the literature [43, 15]. The contact angles of electrospun MIM-GA and functionalized MIM-GA were $134.0 \pm 1.0$ and $125.0 \pm 1.2^{\circ}$, respectively. The hydrophobicity of MIM-GA increased with the presence of GA and decreased with the absence of GA. The contact angle was smaller for functionalized MIM-GA due to the hydrophilic functional groups such as $\mathrm{C}=\mathrm{O}, \mathrm{C}-\mathrm{O}$, and $\mathrm{C}-\mathrm{N}$ of the polymeric matrix (poly(acrylamide)) corresponding

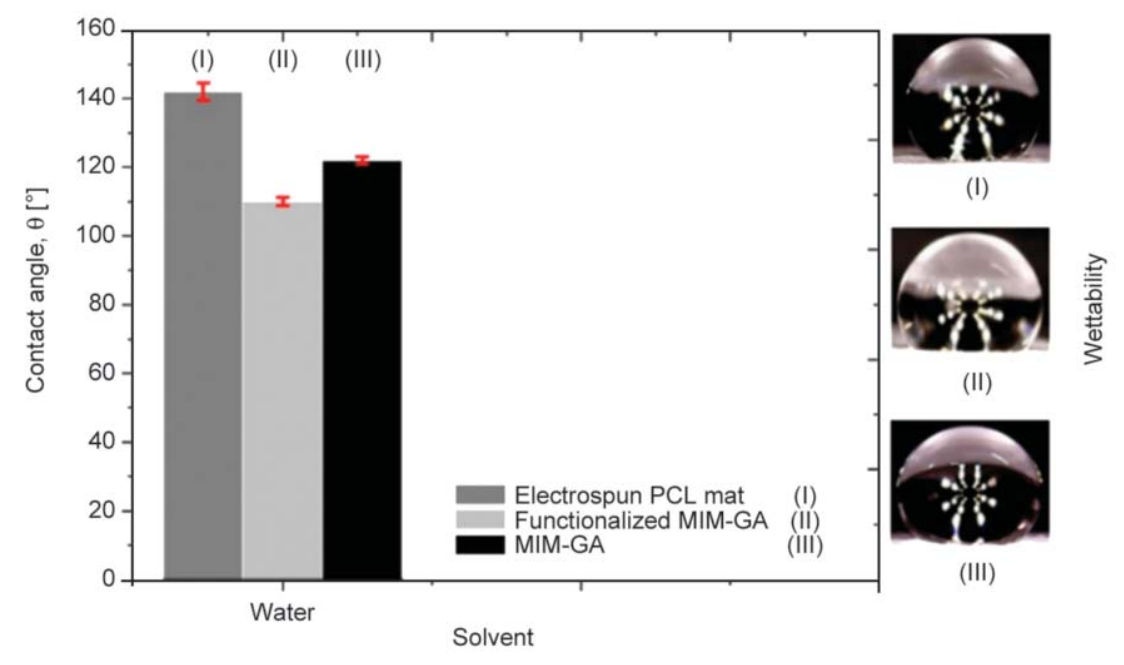

Figure 10. Graph of contact angle of the electrospun MIM-GA and functionalized MIM-GA compared with the electrospun PCL mat. 
to 1726,1044 , and $1454 \mathrm{~cm}^{-1}$ bands, respectively (Table 1). These functional groups interact better with water [15]. On the other hand, the presence of GA occupied the active sites with hydrophilic functional groups of GA, and the hydrophobic group (aromatic ring) may be responsible for the increase in contact angle. Therefore, contact angle measurements can be a technique to qualitatively assess the functionalization of MIM obtained by electrospinning in a simple, practical, and low-cost way.

\section{Conclusions}

A gallic acid imprinted polymer was successfully manufactured by electrospinning. It was possible to observe PCL micrometric fibers formation and the irregular insertion of GA imprinted polymer particles in the material that preserved the integrity of fibers after electrospinning. XPS results showed differences in peak intensity due to the functionalization process, indicating surface changes, while NEXAFS results showed molecular groups that contribute to the electronic transition to anti-bonding $\pi$ orbitals on the polymeric matrices. HPLC corroborated the GA removal from electrospun MIM-GA. Chemical characterization by FTIR confirmed the synthesis of GA imprinted polymer and clearly showed the functionalization of MIM-GA. The contact angle was smaller for functionalized MIM-GA than electrospun MIMGA due to the absence of GA. The GA extraction by functionalized MIM-GA was $96 \pm 1.5 \%$ at immersion time in 150 minutes. Obviously, the electrospun PCL mat cannot extract the GA of the solution. Therefore, the combination of electrospinning and molecular imprinting polymerization techniques proved to be an excellent alternative to produce a membrane with molecular imprinting for the specific detection, extraction, and purification of this important natural polyphenol, GA, with potential application in electrochemical sensors, molecular sieves, and solidphase extraction.

\section{Acknowledgements}

We would like to thank Thematic Laboratory of Microscopy and Nanomaterials-LTMN, CT-INFRA-FINEP, National Council for Scientific and Technological Development CNPq (proc. 308660/2015-3), Coordination for the Improvement of Higher Education Personnel -CAPES, Federal University of Amazonas - UFAM, and Amazonas State Research Support Foundation - FAPEAM (proc. 062.00113/2016) for financial support.

\section{References}

[1] Trotta F., Biasizzo M., Caldera F.: Molecularly imprinted membranes. Membranes, 2, 440-477 (2012).

https://doi.org/10.3390/membranes2030440

[2] Zaidi S. A.: Recent developments in molecularly imprinted polymer nanofibers and their applications. Analytical Methods, 7, 7406-7415 (2015). https://doi.org/10.1039/C5AY01609F

[3] Ye L.: Synthetic strategies in molecular imprinting. in 'Molecularly imprinted polymers in biotechnology' (eds.: Mattiasson B., Ye L.), Springer, Cham, Vol 150, 1-24 (2015). https://doi.org/10.1007/10_2015 313

[4] de Middeleer G., Dubruel P., de Saeger S.: Molecularly imprinted polymers immobilized on 3D printed scaffolds as novel solid phase extraction sorbent for metergoline. Analytica Chimica Acta, 986, 57-70 (2017). https://doi.org/10.1016/j.aca.2017.07.059

[5] Vasapollo G., del Sole R., Mergola L., Lazzoi M. R., Scardino A., Scorrano S., Mele G.: Molecularly imprinted polymers: Present and future prospective. International Journal of Molecular Sciences, 12, 5908-5945 (2011). https://doi.org/10.3390/ijms12095908

[6] Santos M. G., Abrão L. C. C., de Souza Freitas L. A., de Oliveira Isac Moraes G., de Lima M. M., Figueiredo E. C.: Molecularly imprinted polymers in sample preparation for organic compounds analysis: Applications and trends. Scientia Chromatographica, 4, 161-195 (2012). https://doi.org/10.4322/sc.2012.012

[7] Chen L., Wang X., Lu W., Wu X., Li J.: Molecular imprinting: Perspectives and applications. Chemical Society Reviews, 45, 2137-2211 (2016).

https://doi.org/10.1039/C6CS00061D

[8] Pardeshi S., Singh S. K.: Precipitation polymerization: A versatile tool for preparing molecularly imprinted polymer beads for chromatography applications. RSC Advances, 6, 23525-23536 (2016). https://doi.org/10.1039/C6RA02784A

[9] Shah N., Rehan T., Park J. K.: Adsorptive molecularly imprinted composite membranes for chiral separation of phenylalanine. Polish Journal of Chemical Technology, 18, 22-29 (2016).

https://doi.org/10.1515/pjct-2016-0044

[10] Lu Y., Zhao B., Ren Y., Xiao G., Wang X., Li C.: Waterassisted formation of novel molecularly imprinted polymer membranes with ordered porous structure. Polymer, 48, 6205-6209 (2007).

https://doi.org/10.1016/j.polymer.2007.08.045

[11] Ulbricht M., Belter M., Langenhangen U., Schneider F., Weigel W.: Novel molecularly imprinted polymer (MIP) composite membranes via controlled surface and pore functionalizations. Desalination, 149, 293-295 (2002).

https://doi.org/10.1016/s0011-9164(02)00800-7 
[12] Yoshikawa M., Nakai K., Matsumoto H., Tanioka A., Guiver M. D., Robertson G. P.: Molecularly imprinted nanofiber membranes from carboxylated polysulfone by electrospray deposition. Macromolecular Rapid Communications, 28, 2100-2105 (2007).

https://doi.org/10.1002/marc.200700359

[13] Ruggieri F., D’Archivio A. A., Di Camillo D., Lozzi L., Maggi M. A., Mercorio R., Santucci S.: Development of molecularly imprinted polymeric nanofibers by electrospinning and applications to pesticide adsorption. Journal of Separation Science, 38, 1402-1410 (2015). https://doi.org/10.1002/jssc.201500033

[14] Irshad M., Iqbal N., Mujahid A., Afzal A., Hussain T., Sharif A., Ahmad E., Athar M.: Molecularly imprinted nanomaterials for sensor applications. Nanomaterials, 3, 615-637 (2013). https://doi.org/10.3390/nano3040615

[15] Xue J., Wu T., Dai Y., Xia Y.: Electrospinning and electrospun nanofibers: Methods, materials, and applications. Chemical Reviews, 119, 5298-5415 (2019). https://doi.org/10.1021/acs.chemrev.8b00593

[16] Yoshimatsu K., Ye L., Lindberg J., Chronakis I. S.: Selective molecular adsorption using electrospun nanofiber affinity membranes. Biosensors and Bioelectronics, 23, 1208-1215 (2008). https://doi.org/10.1016/j.bios.2007.12.002

[17] Li L., Liu H., Lei X., Zhai Y.: Electrospun nanofiber membranes containing molecularly imprinted polymer (MIP) for rhodamine B (RhB). Advances in Chemical Engineering and Science, 2, 266-274 (2012).

https://doi.org/10.4236/aces.2012.22031

[18] Zaidi S. A.: Recent developments in molecularly imprinted polymer nanofibers and their applications. Analytical Methods, 7, 7406-7415 (2015). https://doi.org/10.1039/c5ay01609f

[19] Chuysinuan P., Chimnoi N., Techasakul S., Supaphol P.: Gallic acid loaded electrospun poly(L-lactic acid) fiber mats and their release characteristic. Macromolecular Chemistry and Physics, 210, 814-822 (2009). https://doi.org/10.1002/macp.200800614

[20] Locatelli C., Filippin-Monteiro F. B., Creczynski-Pasa T. B.: Alkyl esters of gallic acid as anticancer agents: A review. European Journal of Medicinal Chemistry, 60, 233-239 (2013).

https://doi.org/10.1016/j.ejmech.2012.10.056

[21] Gomes C., Sadoyan G., Dias R. C. S., Costa R. P. F. N.: Development of molecularly imprinted polymers to target polyphenols present in plant extracts. Processes, 5, 72/1-72/24 (2017).

https://doi.org/10.3390/pr5040072

[22] Pardeshi S., Dhodapkar R., Kumar A.: Molecularly imprinted microspheres and nanoparticles prepared using precipitation polymerisation method for selective extraction of gallic acid from Emblica officinalis. Food Chemistry, 146, 385-393 (2014).

https://doi.org/10.1016/j.foodchem.2013.09.084
[23] Pardeshi S., Dhodapkar R., Kumar A.: Studies of the molecular recognition abilities of gallic acid-imprinted polymer prepared using a molecular imprinting technique. Adsorption Science and Technology, 30, 23-34 (2012).

https://doi.org/10.1260/0263-6174.30.1.23

[24] Croitoru C., Patachia S.: Molecularly imprinted poly (vinyl alcohol) for the selective absorption of gallic acid from aqueous solutions. Bulletin of the Transilvania University of Brasov: Engineering Sciences, 2, 109-116 (2009).

[25] de Moraes Segundo J. D. P., de Moraes M. O. S., Brito W. R., d'Ávila M. A.: Incorporation of molecularly imprinted polymer nanoparticles in electrospun polycaprolactone fibers. Materials Letters, 275, 128088/1128088/4 (2020). https://doi.org/10.1016/j.matlet.2020.128088

[26] Wagner C. D., Davis L. E., Zeller M. V., Taylor J. A., Raymond R. H., Gale L. H.: Empirical atomic sensitivity factors for quantitative analysis by electron spectroscopy for chemical analysis. Surface and Interface Analysis, 3, 211-225 (1981). https://doi.org/10.1002/sia.740030506

[27] Zubavichus Y., Zharnikov M., Shaporenko A., Fuchs O., Weinhardt L., Heske C., Umbach E., Denlinger J. D., Grunze M.: Soft X-ray induced decomposition of phenylalanine and tyrosine: A comparative study. The Journal of Physical Chemistry A, 108, 4557-4565 (2004).

https://doi.org/10.1021/jp049376f

[28] Schneider C. A., Rasband W. S., Eliceiri K. W.: NIH image to imageJ: 25 years of image analysis. Nature Methods, 9, 671-675 (2012).

https://doi.org/10.1038/nmeth.2089

[29] Dietrich P. M., Glamsch S., Ehlert C., Lippitz A., Kulak N., Unger W. E. S.: Synchrotron-radiation XPS analysis of ultra-thin silane films: Specifying the organic silicon. Applied Surface Science, 363, 406-411 (2016). https://doi.org/10.1016/j.apsusc.2015.12.052

[30] Ali M. A., Srivastava S., Mondal K., Chavhan P. M., Agrawal V. V., John R., Sharma A., Malhotra B. D.: A surface functionalized nanoporous titania integrated microfluidic biochip. Nanoscale, 6, 13958-13969 (2014). https://doi.org/10.1039/c4nr03791j

[31] Shard A. G., Whittle J. D., Beck A. J., Brookes P. N., Bullett N. A., Talib R. A., Mistry A., Barton D., McArthur S. L.: A NEXAFS examination of unsaturation in plasma polymers of allylamine and propylamine. The Journal of Physical Chemistry B, 108, 12472-12480 (2004). https://doi.org/10.1021/jp048250f

[32] Yang Z., Yang Y., Yan W., Tu Q., Wang J., Huang N.: Construction of polyfunctional coatings assisted by gallic acid to facilitate $c o$-immobilization of diverse biomolecules. ACS Applied Materials and Interfaces, 5, 10495-10501 (2013). https://doi.org/10.1021/am403478k 
[33] Solomon D., Lehmann J., Kinyangi J., Liang B., Heymann K., Dathe L., Hanley K., Wirick S., Jacobsen C.: Carbon (1s) NEXAFS spectroscopy of biogeochemically relevant reference organic compounds. Soil Science Society of America Journal, 73, 1817-1830 (2009). https://doi.org/10.2136/sssaj2008.0228

[34] Kaznacheyev K., Osanna A., Jacobsen C., Plashkevych O., Vahtras O., Ågren H., Carravetta V., Hitchcock A. P.: Innershell absorption spectroscopy of amino acids. The Journal of Physical Chemistry A, 106, 3153-3168 (2002). https://doi.org/10.1021/jp013385w

[35] Samuel N. T., Lee C-Y., Gamble L. J., Fischer D. A., Castner D. G.: NEXAFS characterization of DNA components and molecular-orientation of surface-bound DNA oligomers. Journal of Electron Spectroscopy and Related Phenomena, 152, 134-142 (2006). https://doi.org/10.1016/j.elspec.2006.04.004

[36] Ade H., Hitchcock A. P.: NEXAFS microscopy and resonant scattering: Composition and orientation probed in real and reciprocal space. Polymer, 49, 643-675 (2008). https://doi.org/10.1016/j.polymer.2007.10.030

[37] Lee Y., Chang C., Yau S., Fan L., Yang Y., Yang L. O., Itaya K.: Conformations of polyaniline molecules adsorbed on $\mathrm{Au}(111)$ probed by in situ STM and ex situ XPS and NEXAFS. Journal of the American Chemical Society, 131, 6468-6474 (2009). https://doi.org/10.1021/ja809263y
[38] Watts B., Swaraj S., Nordlund D., Lüning J., Ade H.: Calibrated NEXAFS spectra of common conjugated polymers. The Journal of Chemical Physics, 134, 024702/1-024702/15 (2011).

https://doi.org/10.1063/1.3506636

[39] Bhawani S. A., Sen T. S., Ibrahim M. N. M.: Synthesis of molecular imprinting polymers for extraction of gallic acid from urine. Chemistry Central Journal, 12, 19/119/7 (2018).

https://doi.org/10.1186/s13065-018-0392-7

[40] Gorodzha S. N., Surmeneva M. A., Surmenev R. A.: Fabrication and characterization of polycaprolactone cross-linked and highly-aligned 3-D artificial scaffolds for bone tissue regeneration via electrospinning technology. IOP Conference Series: Materials Science and Engineering, 98, 012024/1-012024/6 (2015). https://doi.org/10.1088/1757-899x/98/1/012024

[41] Mohammed-Ziegler I., Billes F.: Vibrational spectroscopic calculations on pyrogallol and gallic acid. Journal of Molecular Structure: THEOCHEM, 618, 259265 (2002). https://doi.org/10.1016/s0166-1280(02)00547-x

[42] Chiem L. T., Huynh L., Ralston J., Beattie D. A.: An in situ ATR-FTIR study of polyacrylamide adsorption at the talc surface. Journal of Colloid and Interface Science, 297, 54-61 (2006). https://doi.org/10.1016/j.jcis.2005.10.037

[43] Yoo H-J., Jwa S-K.: Efficacy of $\beta$-caryophyllene for periodontal disease related factors. Archives of Oral Biology, 100, 113-118 (2019).

https://doi.org/10.1016/j.archoralbio.2019.02.015 